\title{
Contact Detection Approach between Wheel and Rail Surfaces
}

\author{
F. Marques ${ }^{1}$, H. Magalhães ${ }^{2,3}$, J. Pombo $^{2,3,4}$, J. Ambrósio $^{3}$ and P. Flores ${ }^{1}$ \\ ${ }^{1}$ CMEMS-UMinho, Universidade do Minho, Portugal, e-mail: \\ fmarques@dem.uminho.ptpflores@dem.uminho.pt \\ ${ }^{2}$ Institute of Railway Research, School of Computing and Engineering, University \\ ofHuddersfield, UK, e-mail: h.magalhaes@hud.ac.ukj.pombo@hud.ac.uk \\ ${ }^{3}$ IDMEC, Instituto Superior Técnico, Universidade de Lisboa, Portugal, e-mail: \\ jorge.ambrosio@tecnico.ulisboa.pt \\ ${ }^{4}$ ISEL, IPL, Lisboa, Portugal
}

\begin{abstract}
.
This work presents a general formulation to identify the contact points for the interaction between wheels and rails in the context of railway dynamics simulations. This formulation treats the wheel and rail as parametric surfaces and searches the contact between each wheel strip and the rail independently to avoid the numerical difficulties due to the wheel concave zone. This methodology assumes the rail as locally straight and takes advantage that its potential contacting surface is always convex. For the evaluation of contact forces, two Hertzian-based models are employed for normal and creep forces. A trailer vehicle running on a curved track is used to demonstrate the effectiveness of this methodology.
\end{abstract}

Key words: Wheel-Rail Contact, Contact Detection, Multibody Dynamics, Parametric Surfaces

\section{Introduction}

The vehicle-track interaction has a significant impact on the dynamics of railway vehicles, therefore, the computational modeling of wheel-rail contact interaction has been investigated in terms of contact search [1], normal and creep forces evaluation [2], determination of contact patch shape [3], among others. In fact, the development of more accurate and efficient methodologies to evaluate wheel-rail contact improves the reliability and applicability of railway dynamics simulation.

A typical contact detection procedure between wheel and rail involves the geometric definition of both bodies and the identification of the contact location. There are two main methodologies to handle this problem, namely the constraint and the elastic approaches. The former considers the bodies fully rigid, a set of nonlinear kinematic constraints are defined, and the contact forces are determined during the resolution of the equations of motion [4], while the latter allows pene- 
tration between bodies, since they can locally deform, and the normal forces are obtained with a spring-like behavior [5]. The elastic approach is employed here.

Wheel and rail elements are typically defined through parametric surfaces in which their profiles are described by continuous functions. In most cases, their interaction occurs between the rail head and wheel flange or wheel tread, which represent non-conformal configurations. However, in the wheel transition zone, conformal contact can occur, leading to the appearance of numerical problems [6]. Some authors neglect the concave transition zone between tread and flange to avoid these issues [2], however, the results' accuracy may become compromised.

The main objective of this work is to present a methodology for the contact detection between wheel and rail surfaces. The remainder of this paper is divided as follows. Section 2 explains the surfaces parametrization as well as the procedure to identify the contact points. In Section 3, the contact force models utilized here are described. A trailer vehicle running on a curved track is used as example of application in Section 4. Finally, some conclusions are provided in Section 5.

\section{Wheel and Rail Parametrization}

An accurate mathematical description of the wheel and rail contacting surfaces improves the accuracy on the contact detection. Therefore, the rail is obtained through the sweep of its cross-section along a given path, and the wheel is defined by the revolution of its cross-section. Here, both surfaces are parametrized, namely a point on the rail is given by the path's arclength $\left(s_{\mathrm{r}}\right)$ and its lateral coordinate $\left(u_{\mathrm{r}}\right)$, and a point on the wheel surface is defined through the angular parameter $\left(s_{\mathrm{w}}\right)$ and the lateral coordinate $\left(u_{\mathrm{w}}\right)$. This parametrization is schematically represented in Fig. 1. Moreover, both wheel and rail profiles are defined through analytical functions given in the respective standards and represented in Fig. 2.

Two arbitrary points, $P$ and $Q$, located on rail and wheel surfaces can be given as function of the surface parameters. Regarding point $P$, the arclength of the rail $s_{\mathrm{r}, P}^{\text {side }}$ allows to identify the position of a given rail cross-section, $\mathbf{r}_{\mathrm{r}}^{\text {side }}$, and its orientation by a set of vectors $\mathbf{t}_{\mathrm{r}}^{\text {side }}, \mathbf{n}_{\mathrm{r}}^{\text {side }}$ and $\mathbf{b}_{\mathrm{r}}^{\text {side }}$, which represent the tangent, normal and binormal vectors, respectively, as depicted in Fig 1. Thus, the rail transformation matrix can be defined as

$$
\mathbf{A}_{\mathrm{r}}^{\text {side }}=\left[\begin{array}{lll}
\mathbf{t}_{\mathrm{r}}^{\text {side }} & \mathbf{n}_{\mathrm{r}}^{\text {side }} & \mathbf{b}_{\mathrm{r}}^{\text {side }}
\end{array}\right]
$$

Hence, the position of point $P$ is calculated as

$$
\mathbf{r}_{P}^{\text {side }}=\mathbf{r}_{\mathrm{r}}^{\text {side }}+\mathbf{A}_{\mathrm{r}}^{\text {side }}\left\{\begin{array}{lll}
0 & u_{\mathrm{r}, P}^{\text {side }} & f_{\mathrm{r}, P}^{\text {side }}
\end{array}\right\}^{\mathrm{T}}
$$

where $u_{\mathrm{r}, P}^{\text {side }}$ is the rail lateral parameter of point $P$, and $f_{\mathrm{r}, P}^{\text {side }}$ denotes the ordinate of the rail profile for $u_{\mathrm{r}, P}^{\text {side }}$, as shown in Fig. 2 (left). Moreover, the superscript side stands for "L" or "R", whether it refers to the left or right elements, respectively. 


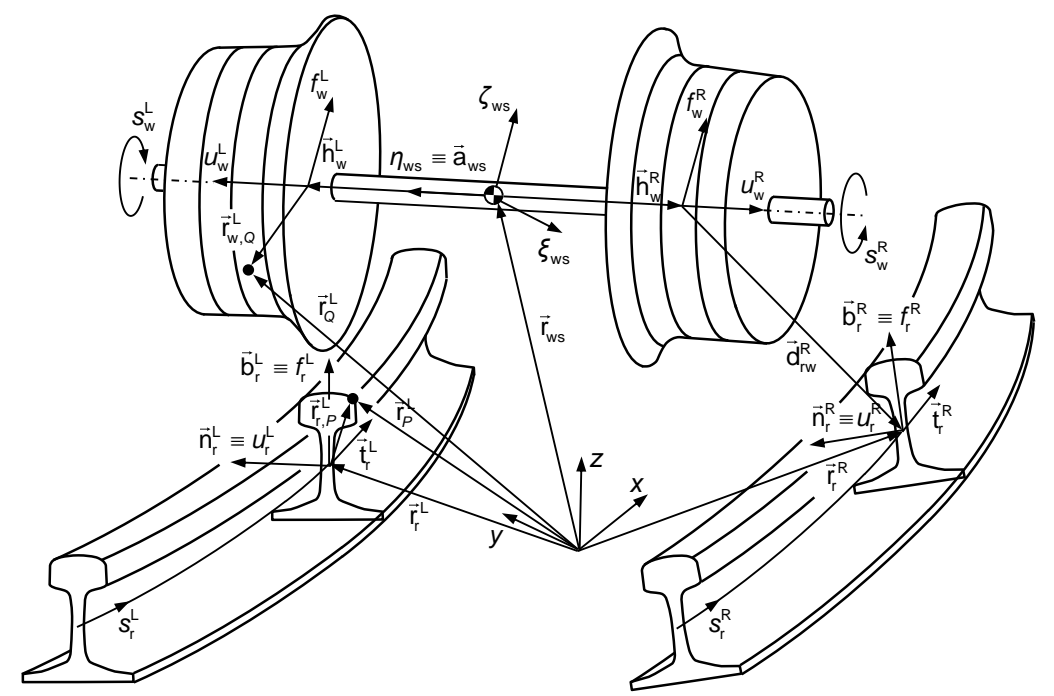

Fig. 1 Parametrization of wheel and rail surfaces
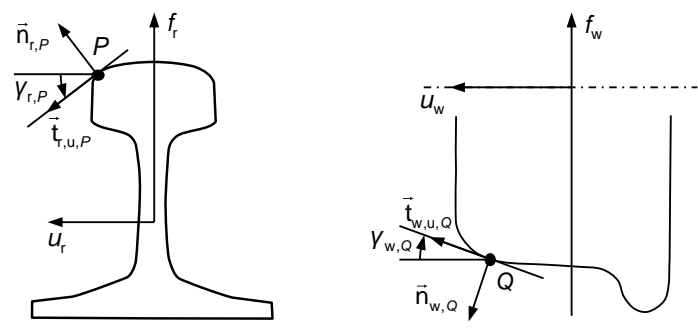

Fig. 2 Representation of both rail and wheel profiles and their local coordinate system.

In order to obtain the rail position and orientation to be used for the contact detection, it must be found the parameter $s_{\mathrm{r}}^{\text {side }}$ that minimizes the distance between the wheel and rail profiles represented by $\mathbf{d}_{\mathrm{rw}}^{\text {side }}$, shown in Fig 1., and calculated as

$$
\mathbf{d}_{\mathrm{rw}}^{\text {side }}=\mathbf{r}_{\mathrm{r}}^{\text {side }}-\left(\mathbf{r}_{\mathrm{ws}}+\mathbf{h}_{\mathrm{w}}^{\text {side }}\right)
$$

in which $\mathbf{r}_{\mathrm{ws}}$ defines the location of wheelset mass center and $\mathbf{h}_{\mathrm{w}}^{\text {side }}$ is the relative position between the wheelset mass center and the wheel profile origin given as

$$
\mathbf{h}_{\mathrm{w}}^{\mathrm{L}}=H / 2 \cdot \mathbf{a}_{\mathrm{ws}} \quad \text { or } \quad \mathbf{h}_{\mathrm{w}}^{\mathrm{R}}=-H / 2 \cdot \mathbf{a}_{\mathrm{ws}}
$$

where $H$ denotes the distance between both wheel profiles and $\mathbf{a}_{\mathrm{ws}}$ is the wheelset axial unit vector. The minimum distance can be obtained by satisfying 


$$
\mathbf{d}_{\mathrm{rw}}^{\text {side }} \cdot \mathbf{t}_{\mathrm{r}}^{\text {side }}=0
$$

Hence, $s_{\mathrm{r}}^{\text {side }}$ is obtained and utilized to determine the rail profile location, $\mathbf{r}_{\mathrm{r}}^{\text {side }}$, and orientation, $\mathbf{A}_{\mathrm{r}}^{\text {side }}$, for the remaining contact search. In what concerns to point Q on the wheel surface, it is defined following the representation of Fig. 1 as

$$
\mathbf{r}_{Q}^{\text {side }}=\mathbf{r}_{\mathrm{ws}}+\mathbf{h}_{\mathrm{w}}^{\text {side }}+\mathbf{A}_{\mathrm{w}}^{\text {side }} \mathbf{A}_{\mathrm{w}, \mathrm{s}}^{\text {side }}\left\{\begin{array}{lll}
0 & u_{\mathrm{w}, Q}^{\text {side }} & f_{\mathrm{w}, Q}^{\text {side }}
\end{array}\right\}^{\mathrm{T}}
$$

in which $u_{\mathrm{w}, Q}^{\text {side }}$ is the lateral wheel surface parameter of point $Q, f_{\mathrm{w}, Q}^{\text {side }}$ denotes the ordinate of the wheel profile function for the position $u_{\mathrm{w}, Q}^{\text {side }}$, as represented in Fig. 2 (right), and $\mathbf{A}_{\mathrm{w}}^{\text {side }}$ is the wheel transformation matrix which allows to obtain a wheel coordinate system aligned with the rail frame, and it can be computed as

$$
\mathbf{A}_{\mathrm{w}}^{\text {side }}=\left[\begin{array}{lll}
\mathbf{n}_{\mathrm{w}}^{\text {side }} & \mathbf{a}_{\mathrm{w}}^{\text {side }} & \mathbf{b}_{\mathrm{w}}^{\text {side }}
\end{array}\right] \text { in which }\left\{\begin{array}{l}
\mathbf{a}_{\mathrm{w}}^{\text {side }}=\mathbf{a}_{\mathrm{ws}} \\
\mathbf{b}_{\mathrm{w}}^{\text {side }}=\mathbf{t}_{\mathrm{r}}^{\text {side }} \times \mathbf{a}_{\mathrm{w}}^{\text {side }} / \| \mathbf{t}_{\mathrm{r}}^{\text {side }} \times \mathbf{a}_{\mathrm{w}}^{\text {side } \|} \\
\mathbf{n}_{\mathrm{w}}^{\text {side }}=\mathbf{a}_{\mathrm{w}}^{\text {side }} \times \mathbf{b}_{\mathrm{w}}^{\text {side }}
\end{array}\right.
$$

The transformation matrix $\mathbf{A}_{\mathrm{w}, \mathrm{s}}^{\text {side }}$ specifies the rotation around the wheel axis, which depends on the angular surface parameter, $s_{\mathrm{w}, Q}^{\text {side }}$, and is defined as

$$
\mathbf{A}_{\mathrm{w}, \mathrm{s}}^{\mathrm{L}}=\left[\begin{array}{ccc}
\cos \left(s_{\mathrm{w}, Q}^{\mathrm{L}}\right) & 0 & \sin \left(s_{\mathrm{w}, Q}^{\mathrm{L}}\right) \\
0 & 1 & 0 \\
-\sin \left(s_{\mathrm{w}, Q}^{\mathrm{L}}\right) & 0 & \cos \left(s_{\mathrm{w}, Q}^{\mathrm{L}}\right)
\end{array}\right] \text { or } \quad \mathbf{A}_{\mathrm{w}, \mathrm{s}}^{\mathrm{R}}=\left[\begin{array}{ccc}
-\cos \left(s_{\mathrm{w}, Q}^{\mathrm{R}}\right) & 0 & -\sin \left(s_{\mathrm{w}, Q}^{\mathrm{R}}\right) \\
0 & -1 & 0 \\
-\sin \left(s_{\mathrm{w}, Q}^{\mathrm{R}}\right) & 0 & \cos \left(s_{\mathrm{w}, Q}^{\mathrm{R}}\right)
\end{array}\right]
$$

Based on the relation between wheel and rail coordinate systems, the roll angle, $\varphi$, and yaw angle, $\alpha$, are obtained through successive rotations of vector $\mathbf{a}_{\mathrm{w}}^{\text {side }}$. In order to find the potential contact points, one constraint concerns the parallelism between both normal vectors. Considering the rail is locally straight, the normal vector to the wheel surface must be in the plane formed by $\mathbf{n}_{\mathrm{r}}^{\text {side }}$ and $\mathbf{b}_{\mathrm{r}}^{\text {side }}$, which yields that the first element of the wheel normal vector in the rail coordinate system must be zero. This vector is obtained from the following consecutive rotations

$$
\mathbf{n}_{\mathrm{w}, Q, \mathrm{r}}^{\text {side }}=\mathbf{R}_{\varphi}^{\text {side }} \mathbf{R}_{\alpha}^{\text {side }} \mathbf{A}_{\mathrm{w}, \mathrm{s}}^{\text {side }} \mathbf{n}_{\mathrm{w}, Q, \mathrm{w}}^{\text {side }}
$$

in which $\mathbf{R}_{\varphi}^{\text {side }}$ and $\mathbf{R}_{\alpha}^{\text {side }}$ are the rotation matrices of roll and yaw angles, respectively, and the normal vector to the wheel surface in local coordinates is given as

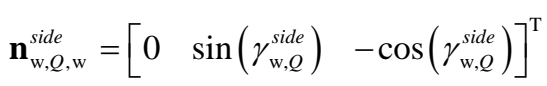

where $\gamma_{\mathrm{w}, Q}^{\text {side }}$ denotes the wheel contact angle on point $\mathrm{Q}$, which is represented in Fig. 2, and can be calculated as function of the profile function as 


$$
\gamma_{\mathrm{w}, Q}^{\text {side }}=\arctan \left(f_{\mathrm{w}, Q}^{\prime \text { side }}\right)
$$

It must be noted that the remaining vectors represented in Fig. 2 can be similarly obtained. Thus, imposing that the first element of the $\mathbf{n}_{\mathrm{w}, Q, \mathrm{r}}^{\text {side }}$ is zero, it yields

$$
s_{\mathrm{w}, Q}^{\text {side }}=\arcsin \left(-\tan \left(\alpha^{\text {side }}\right) f_{\mathrm{w}, Q}^{\prime \text { side }}\right)
$$

Moreover, the distance vector between wheel and rail potential contact points, $\mathbf{d}^{\text {side }}$, must also be parallel to both normal vectors. Thus, it is possible to calculate the increment of location of rail contact point so $\mathbf{d}^{\text {side }}$ also falls in the plane formed by $\mathbf{n}_{\mathrm{r}}^{\text {side }}$ and $\mathbf{b}_{\mathrm{r}}^{\text {side }}$, as depicted in Fig. 3. This increment is determined as

$$
\Delta x^{\mathrm{L}}=\left(\left|f_{\mathrm{w}, Q}^{\mathrm{L}}\right| f_{\mathrm{w}, Q}^{\mathrm{L}}-u_{\mathrm{w}, Q}^{\mathrm{L}}\right) \sin \left(\alpha^{\mathrm{L}}\right) \quad \text { or } \quad \Delta x^{\mathrm{R}}=\left(u_{\mathrm{w}, Q}^{\mathrm{R}}-\left|f_{\mathrm{w}, Q}^{\mathrm{R}}\right| f_{\mathrm{w}, Q}^{\prime \mathrm{R}}\right) \sin \left(\alpha^{\mathrm{R}}\right)
$$

Accordingly, Eq.(2) must be replaced by

$$
\mathbf{r}_{P}^{\text {side }}=\mathbf{r}_{\mathrm{r}}^{\text {side }}+\mathbf{A}_{\mathrm{r}}^{\text {side }}\left\{\Delta x^{\text {side }} \quad u_{\mathrm{r}, P}^{\text {side }} \quad f_{\mathrm{r}, P}^{\text {side }}\right\}^{\mathrm{T}}
$$
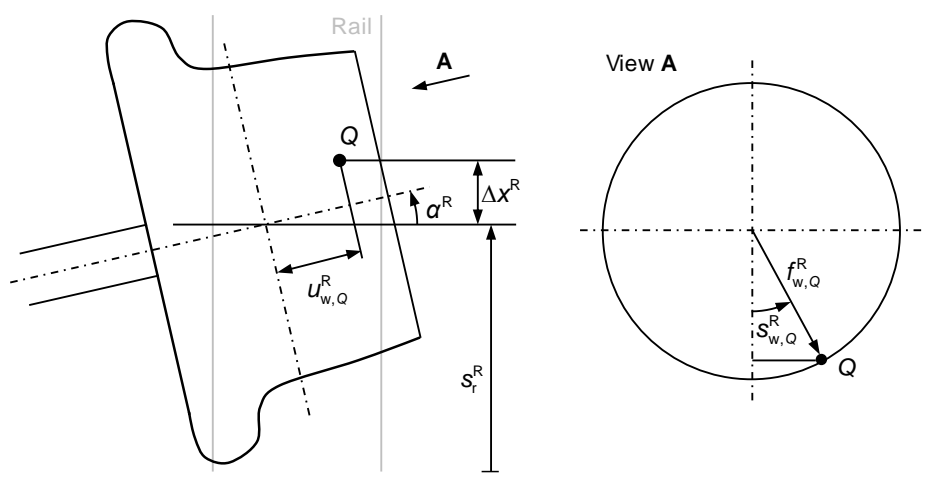

Fig. 3 Representation of the increment on longitudinal direction for the contact point in the rail.

The wheel angular coordinate and the longitudinal position along the rail can be analytically evaluated with Eq. (12) and (13), respectively. Thus, the contact points only depend on $u_{\mathrm{w}, Q}^{\text {side }}$ and $u_{\mathrm{r}, P}^{\text {side }}$. For each wheel strip, the rail lateral parameter which ensures that $\mathbf{d}^{\text {side }}$ and $\mathbf{n}_{\mathrm{r}, P}^{\text {side }}$ are parallel can be obtained by solving

$$
\mathbf{d}^{\text {side }} \cdot \mathbf{t}_{\mathrm{r}, \mathrm{u}, P}^{\text {side }}=0
$$

Therefore, to find the contact points, an optimization problem must be formulated to find the wheel strip with the largest penetration which is given as

$$
\delta=\left\|\mathbf{d}^{\text {side }}\right\| \quad \text { if } \quad \mathbf{n}_{\mathrm{r}, P}^{\text {side }} \cdot \mathbf{d}^{\text {side }}<0
$$




\section{Contact Forces Models}

The contact forces play a key role on the dynamic behavior of the multibody systems, since they must consider the geometrical and physical properties of the contacting bodies and contribute for the stable resolution of the equations of motion.

Regarding the normal contact force evaluation, a Hertzian-based model is utilized here [7] which can be expressed as

$$
f_{\mathrm{n}}= \begin{cases}K \delta^{n} c_{\mathrm{e}} & \dot{\delta} \leq-v_{0} \\ K \delta^{n}\left[c_{\mathrm{e}}+\left(1-c_{\mathrm{e}}\right)\left(3 r^{2}-2 r^{3}\right)\right] & -v_{0}<\dot{\delta}<v_{0} \quad \text { where } \quad r=\frac{\dot{\delta}+v_{0}}{2 v_{0}} \\ K \delta^{n} & \dot{\delta} \geq v_{0}\end{cases}
$$

where $K$ is the contact stiffness that depends on the local geometric and material properties, $\delta$ denotes the penetration depth, $\dot{\delta}$ represents the penetration velocity, $v_{0}$ denotes the tolerance for the penetration velocity, $c_{\mathrm{e}}$ is the coefficient of restitution, and $n$ is an exponent that defines the degree of nonlinearity.

In what concerns to the creep forces, Polach method is adopted in this work since it complies with Hertz theory by considering an elliptical contact patch [8]. The longitudinal and lateral creep forces and spin creep moment are evaluated as

$$
f_{x}=f \frac{v_{x}}{v_{C}}, \quad f_{y}=f \frac{v_{y}}{v_{C}}+f_{y \mathrm{~S}} \frac{\phi}{v_{C}}, \quad m_{z}=0
$$

where $v_{x}, v_{y}$ and $\phi$ are the longitudinal, lateral and spin creepages, respectively, $v$ and $v_{C}$ denote the magnitude of translational creepage and the modified translational creepage, $f$ is the tangential contact force caused by the longitudinal and spin creepages and $f_{y S}$ expresses the lateral tangential force due to spin creepage.

Both forces act as external forces on the wheelsets and are included on the equations of motion which, for constrained multibody systems, can be given as [9]

$$
\left[\begin{array}{cc}
\mathbf{M} & \boldsymbol{\Phi}_{\mathbf{q}}^{T} \\
\boldsymbol{\Phi}_{\mathbf{q}} & \mathbf{0}
\end{array}\right]\left\{\begin{array}{l}
\ddot{\mathbf{q}} \\
\boldsymbol{\lambda}
\end{array}\right\}=\left\{\begin{array}{c}
\mathbf{g} \\
\boldsymbol{\gamma}-2 \alpha \dot{\boldsymbol{\Phi}}-\beta^{2} \mathbf{\Phi}
\end{array}\right\}
$$

in which $\mathbf{M}$ denotes the global mass matrix, $\boldsymbol{\Phi}_{\mathbf{q}}$ expresses the Jacobian matrix of the constraints equations, $\ddot{\mathbf{q}}$ is the generalized accelerations, $\boldsymbol{\lambda}$ denotes the vector of Lagrange multipliers, which represent the reaction forces on ideal joints, $\mathbf{g}$ is the vector of external generalized forces, $\gamma$ is the right-hand side vector of acceleration constraint equations, $\boldsymbol{\Phi}$ and $\dot{\boldsymbol{\Phi}}$ denote the violation of constraints at position and velocity level, respectively, $\alpha$ and $\beta$ are feedback control parameters of Baumgarte technique for constraints stabilization. Eq. (19) must be solved in each time step to get the system's accelerations, which are then integrated over time. 


\section{Example of Application}

A trailer vehicle negotiating a left curve with a radius of $300 \mathrm{~m}$ is used as application case. This multibody model includes 11 bodies, namely 4 wheelsets, 4 axleboxes, 2 bogie frames and the carbody. The vehicle starts with a forward velocity of $18.3 \mathrm{~m} / \mathrm{s}$, being the total simulation time $41 \mathrm{~s}$. The initial configuration considers a lateral misalignment of $2 \mathrm{~mm}$ to promote the hunting motion of the system.
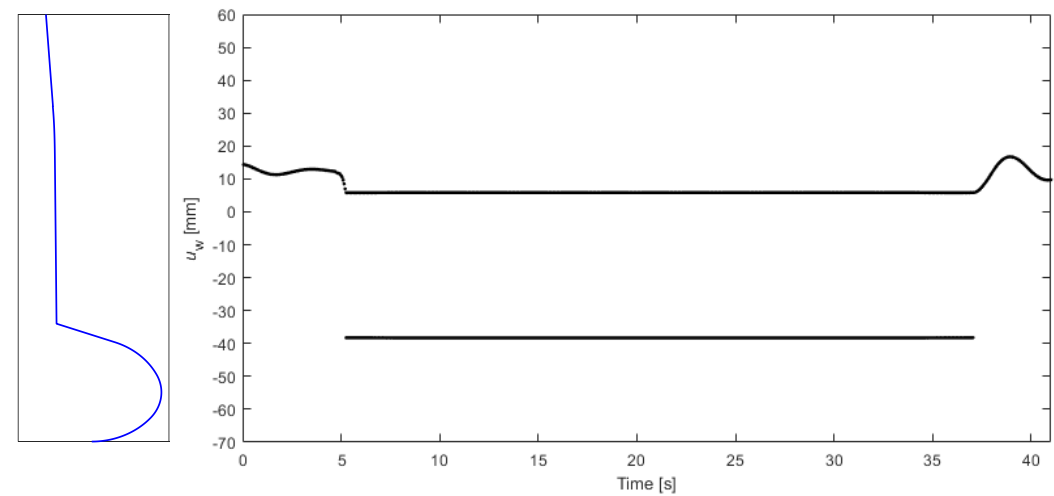

(a)
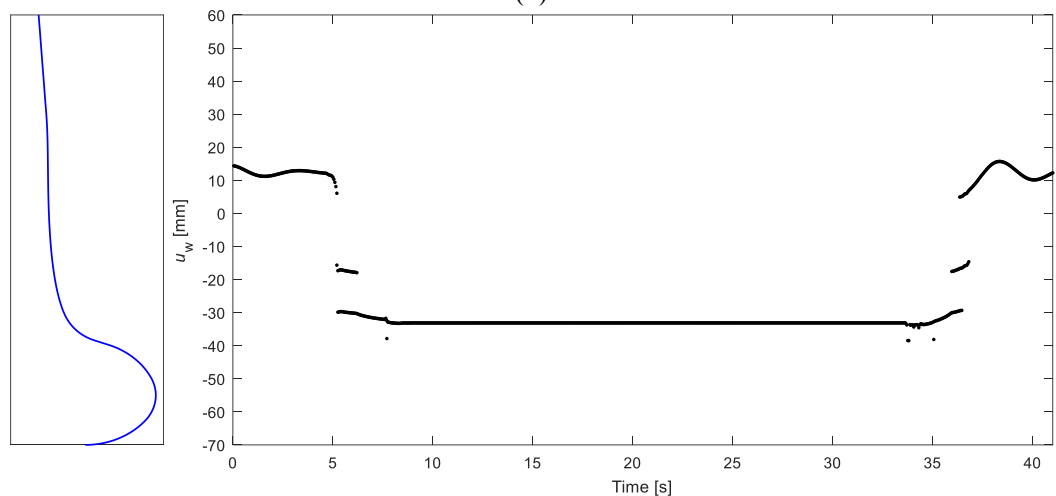

(b)

Fig. 4 Representation of the location of the main point of contact for each patch over time.

The contact in the right wheel of the leading wheelset of the front bogie is analyzed in detail, since it is the external wheel relatively to the curve where the flange contact occurs. Thus, Figs. 4 (a) and (b) display the location of the several contact points in the wheel profile over time for the simplified profile and real profiles, respectively. For the beginning of motion, it is verified that both profiles perform a hunting motion due to the initial misalignment. This can be seen through the wave-like behavior in the contact location. During the curve negotiation, bigger differences are identified. With the simplified profiles, the contact is deter- 
mined independently in the tread and flange, and it is observed that there is simultaneous contact in the tread and flange from $5.2 \mathrm{~s}$ until $37.1 \mathrm{~s}$. On the other hand, for real profiles, during most of the curve, only one contact patch exists, which is in the wheel transition zone. However, in some periods, the wheel and rail may interact in more than one location which results in multiple contact patches.

\section{Conclusions}

A methodology for contact detection between wheel and rail considering realistic geometries is presented here. This method uses the assumption that the rail is locally straight and searches the strip which corresponds to the maximum virtual penetration for each contact patch. The dynamic simulation of a trailer vehicle running on a curved track verifies the effectiveness of the proposed methodology. The results show that this approach allows the existence of multiple contact patches in each wheel-rail pair and that the simplification of the wheel profile in the transition zone does not allow an accurate identification of the contact points.

Acknowledgments The first author is supported by the Portuguese Foundation for Science and Technology (FCT) under grant $\mathrm{PD} / \mathrm{BD} / 114154 / 2016$. This work is also supported by FCT with the reference project UIDB/04436/2020.

\section{References}

1. Marques, F., Magalhães, H, Pombo, J., Ambrósio, J. and Flores, P.: A three-dimensional approach for contact detection between realistic wheel and rail surfaces for improved railway dynamic analysis. Mechanism and Machine Theory, 149, 103825 (2020)

2. Magalhães H., Marques, F., Liu, B., Antunes, P., Pombo, J., Flores, P., Ambrósio, J., Piotrowski, J. and Bruni, S.: Implementation of a non-Hertzian contact model for railway dynamic application. Multibody System Dynamics, 48 (1), 41-78 (2020)

3. Sun, Y., Zhai, W. and Guo, Y.: A robust non-Hertzian contact method for wheel-rail normal contact analysis. Vehicle System Dynamics, 56(12), 1899-1921 (2018)

4. Shabana, A.A., Tobaa, M., Sugiyama, H. and Zaazaa, K.E.: On the Computer Formulations of the Wheel/Rail Contact Problem. Nonlinear Dynamics, 40 (2), 169-193 (2005)

5. Malvezzi, M., Meli, E., Falomi, S. and Rindi, A.: Determination of wheel-rail contact points with semianalytic methods. Multibody System Dynamics, 20, 327-358 (2008)

6. Marques, F., Magalhães, H., Ambrósio, J. and Flores, P.: Approach for conformal contact detection for wheel-rail interaction. In: 7th European Conference on Mechanism Science - EuCoMeS 2018, Aachen, Germany. Mechanisms and Machine Science, 59, $71-78$ (2019)

7. Machado, M., Moreira, P., Flores, P., Lankarani, H.M.: Compliant contact force models in multibody dynamics: Evolution of the Hertz contact theory. Mechanism and Machine Theory, 53, 99-121 (2012)

8. Polach, O.: A Fast Wheel-Rail Forces Calculation Computer Code. Vehicle System Dynamics, Sup. 33, 728-739 (1999)

9. Nikravesh, P.E.: Computer Aided Analysis of Mechanical Systems. Prentice Hall, Englewood Cliffs, New Jersey (1988) 